\title{
Medical assessment of fitness to dive after COVID-19
}

\author{
Jarosław Krzyżak ${ }^{1}$, Krzysztof Korzeniewski ${ }^{2,3}$ \\ ${ }^{1}$ Polish Society of Hyperbaric Medicine and Technology, Gdynia, Poland \\ ${ }^{2}$ Department of Epidemiology and Tropical Medicine, Military Institute of Medicine, Warsaw, Poland \\ ${ }^{3}$ Department of Tropical Medicine and Epidemiology, Institute of Maritime and Tropical Medicine, \\ Medical University of Gdansk, Poland
}

\begin{abstract}
Since the global pandemic caused by the severe acute respiratory syndrome coronavirus 2 (SARS-CoV-2), the etiological agent of a coronavirus disease 2019 (COVID-19) infection started, millions of people globally have become infected. It must not be forgotten that thousands of those affected are commercial or recreational divers. A severe COVID-19 seriously affects the respiratory system and the pulmonary parenchyma but it may also involve the cardiovascular and the blood coagulation system. For this reason, many divers and hyperbaric medicine specialists are becoming increasingly concerned about a diver's ability to safely return to diving after a SARS-CoV-2 infection. The article presents current guidelines for diving fitness health assessment after a COVID-19 both for active and prospective divers, with particular emphasis on the types of diagnostic procedures and tests to be performed in cases of asymptomatic infection with SARS-CoV-2 as well as in patients who have recovered from a symptomatic COVID-19.
\end{abstract}

(Int Marit Health 2021; 72, 3: 223-227)

Key words: diving, fitness, health assessment, COVID-19, recommendations

\section{INTRODUCTION}

It has been over a year since the coronavirus disease 2019 (COVID-19) pandemic broke out, but so far there have been no in-depth population-based studies on long-term sequelae of the disease, especially with respect to divers. However, a better understanding of clinical and radiological signs and symptoms of the disease allowed researchers to formulate a list of guidelines for medical assessment of divers who recovered from a COVID-19 infection in order to evaluate whether or not they can safely return to scuba diving. Understanding the pathophysiology of COVID-19 is critical to ensuring diving safety; the infection does not only increase the risk of pulmonary barotrauma and arterial gas embolism, but it also raises the susceptibility to decompression sickness or pulmonary edema due to cardiological, pulmonary and hematological changes. In fact, the ongoing COVID-19 pandemic may contribute to improving the quality of diving fitness health assessment [1-4].
The regulations applicable to commercial diving are clear: it is within the competence of an employer to refer a diver who has had a COVID-19 infection for fitness to dive re-assessment. The employer is legally bound to refer their employee for periodic examination by an occupational health physician certified as a diving doctor in order to identify or reduce any potential risk of sequelae associated with the severe acute respiratory syndrome coronavirus 2 (SARS-CoV-2) infection and, if there are no contraindications, to issue a certificate of medical fitness to dive. Regrettably, there is no such requirement for recreational divers. Recreational divers who tested positive for COVID-19 should be recommended consult a competent specialist out of concern for their own safety.

\section{RESPIRATORY COMPLICATIONS OF COVID-19}

Symptomatic SARS-CoV-2 infection and a COVID-19 manifest as a typical upper respiratory tract infection; in more

Prof. Krzysztof Korzeniewski, MD, PhD, Military Institute of Medicine, Head of the Department of Epidemiology and Tropical Medicine, Szaserów St. 128, 04-141 Warsaw, $\triangle$ Poland, e-mail: kkorzeniewski@wim.mil.pl; Medical University of Gdansk, Institute of Maritime and Tropical Medicine, Department of Tropical Medicine and Epidemiology,

Powstania Styczniowego St. 9B, 81-519 Gdynia, Poland, e-mail: kkorzeniewski@gumed.edu.pl

This article is available in open access under Creative Common Attribution-Non-Commercial-No Derivatives 4.0 International (CC BY-NC-ND 4.0) license, allowing to download articles and share them with others as long as they credit the authors and the publisher, but without permission to change them in any way or use them commercially. 
severe cases the infection progresses into a bilateral viral pneumonia often leading to death. Lung damage occurs due to a massive inflammatory reaction coupled with the injury caused by assisted mechanical ventilation, high airway pressure and exposure to hyperoxia [3, 4].

A chest X-ray or a computed tomography (CT) scan typically show ground-glass opacities, crazy paving pattern or peripheral (subpleural) parenchymal densities. Bilateral lesions are most pronounced on the $10^{\text {th }}$ day of onset, and then they gradually disappear. The crazy paving pattern tends to resolve after approximately 4 weeks of onset, but the ground-glass opacities are likely to persist for a longer period. During the initial stage of the infection, 15-20\% patients have normal chest radiogram; however, as the infection progresses, peripherally distributed multifocal densities and opacities, air space opacification (cavitation) and lymphadenopathy tend to occur. Radiograms performed at a later stage of the infection often reveal progressing pulmonary fibrosis. A severe COVID-19 infection leaves permanent radiological changes on a chest X-ray $[5,6]$.

It may be assumed that the long-term effects of COVID-19 will resemble the much better-studied complications of the SARS-CoV-1 infection. Approximately $20 \%$ of patients, who recovered from a SARS-CoV-1 infection, had residual lesions on a chest X-ray and abnormal results of pulmonary function tests(Figs. 1,2) 3 weeks after recovery. The patients also complained of general weakness, decreased exercise tolerance, shortness of breath and breathing difficulties. It was found that in patients who have recovered from a COVID-19 infection abnormalities revealed by pulmonary function tests appear to be a more sensitive indicator of lung damage than CT scanning [7]. Even as long as 140 days after the onset of first symptoms, some patients had persistent abnormalities on their chest CT scan, including opacities, reticulation and air trapping (Figs. 3, 4) [3, 6].

If a COVID-19 infection turns out to have a similar course, we might expect that it will potentially have some negative effects on divers' physical health, especially their exercise capacity. The load to the respiratory system during a dive, i.e. under hyperbaric conditions is obviously different from the respiratory load experienced in normal conditions, which is associated with increased breathing resistance of the regulator, the density of breathing gases and the depth of immersion. For this reason, in order to be able to safely return to scuba diving after a COVID-19 infection, a diver will have to demonstrate good exercise capacity. Also, it should be remembered that residual parenchymal lesions such as scars, calcifications, blebs or air trapping may be associated with an increased risk of a pulmonary barotrauma [8]. Additionally, the lesions which have developed in the lung parenchyma and pulmonary vessels can facilitate the transfer of asymptomatic gas embolism to the arterial part of the circulatory system.

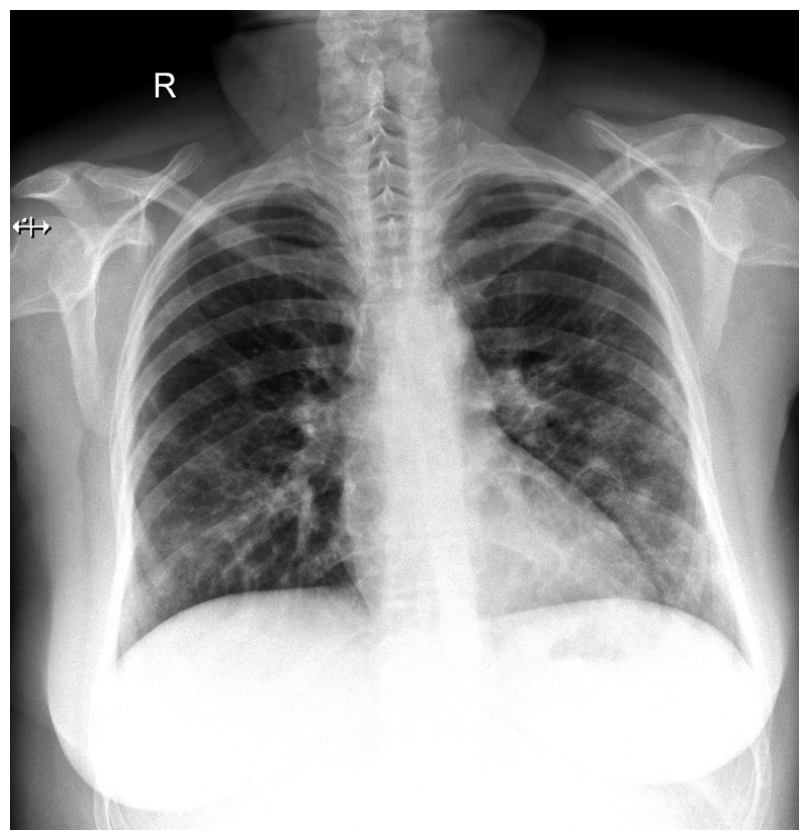

Figure 1. An X-ray image showing pulmonary lesions in mild COVID-19 (foci of peripheral parenchymal densities). Source: X-ray Laboratory at the St. Vincent de Paul Hospital in Gdynia

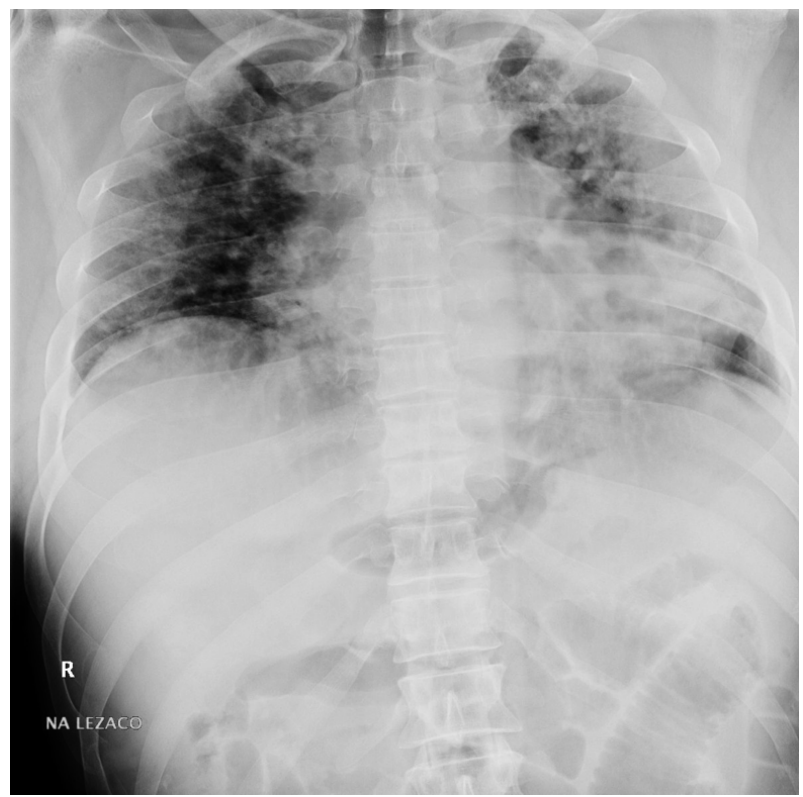

Figure 2. An X-ray image showing pulmonary lesions in severe COVID-19 (bilateral foci of massive and consolidated parenchymal densities). Source: X-ray Laboratory at the St. Vincent de Paul Hospital in Gdynia

\section{CIRCULATORY COMPLICATIONS OF COVID-19}

Although a COVID-19 infection manifests primarily as pneumonia, increasing data suggest that cardiac involvement is also characteristic for the disease. So far, no cardiovascular symptoms have been identified as specific for 


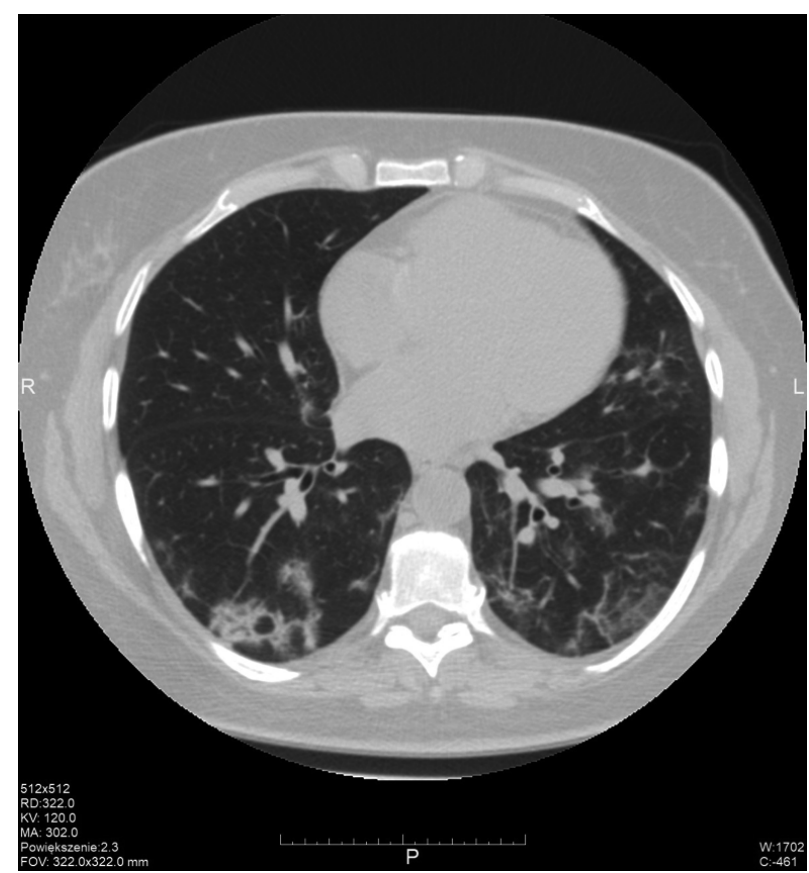

Figure 3. A chest computed tomography scan showing lesions in mild COVID-19 (foci of small peripheral parenchymal densities). Source: X-ray Laboratory at the St. Vincent de Paul Hospital in Gdynia

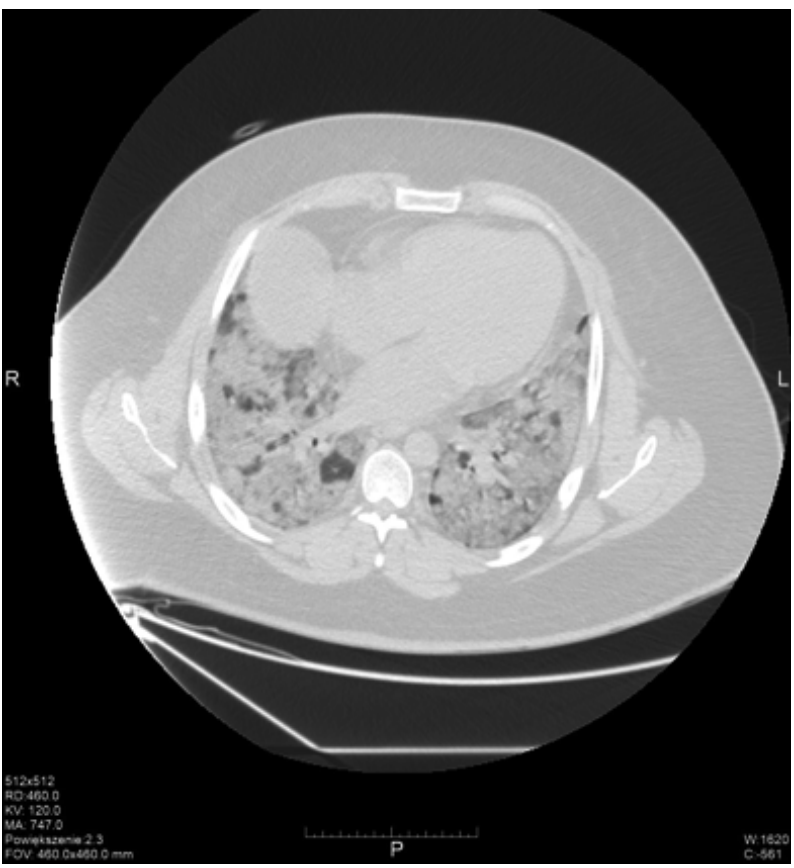

Figure 4. A chest computed tomography scan showing lesions in severe COVID-19 (near complete involvement of the lung parenchyma, ground-glass opacity). Source: X-ray Laboratory at the St. Vincent de Paul Hospital in Gdynia

a COVID-19 infection. Cardiovascular involvement which occurs in the course of infection covers a wide spectrum of conditions including isolated increase in cardiac biomarkers, acute coronary syndrome, arrhythmias, myocarditis and pericarditis [1, 3]. Post-mortem examinations revealed that heart damage was not directly attributable to SARS-CoV-2, but rather that it was secondary to systemic inflammation and hypercoagulable states which occurred in the course of the infection. The virus has, however, been found in the vascular endothelium, suggesting direct invasion. Between $8 \%$ and $28 \%$ of the infected individuals showed elevated cardiac biomarkers - troponin and B-type natriuretic peptide, which was associated with higher mortality from COVID-19 [1, 9].

Both commercial and recreational diving will require a work rate of 6-7 MET (which is approximately $21 \mathrm{~mL}$ $\mathrm{O}_{2} / \mathrm{kg} / \mathrm{min}$ ), or higher in typical emergency situations. Currently, the guidelines recommend at least $10 \mathrm{MET}$ for commercial divers (approximately $35 \mathrm{~mL} \mathrm{O}_{2} / \mathrm{kg} / \mathrm{min}$ ) and $6 \mathrm{MET}$ for recreational divers. It is advisable, however, that divers should be required to reach the rate of $13 \mathrm{MET}$; this would be a good reserve in case of an emergency [2]. It is important that divers who had recovered from a COVID-19 infection undergo a thorough cardiological evaluation. Apart from electrocardiogram and echocardiogram, an exercise tolerance test to estimate maximum oxygen consumption should be performed. Oligosymptomatic cardiac inflammation could be unmasked by a higher physical effort and the stress associated with diving, thus resulting in dangerous arrhythmias or decompensated heart failure [2, 4].

\section{THROMBOEMBOLIC COMPLICATIONS OF COVID-19}

Coronavirus disease, unlike other conditions of a similar type, was found to significantly increase the susceptibility to venous thromboembolism. COVID-19 has been widely reported to cause hypercoagulable states and elevated levels of D-dimers and troponins, which are associated with a much worse prognosis. In its acute phase, the disease may induce a number of thromboembolic complications as well as acute coronary syndrome [3]. In light of the above, the question arises whether or not the hypercoagulable state would predispose a diver who had COVID-19 to decompression sickness, which is considered a generalized inflammatory and pro-thrombotic condition. It seems unlikely that the hypercoagulable state could persist after the acute phase of the disease is over and when a patient has fully recovered from the infection [4].

\section{CURRENT RECOMMENDATIONS FOR DIVERS AFTER COVID-19}

According to the current guidelines, a person cannot be medically cleared to scuba dive if they have had a cough, shortness of breath, difficulty breathing, fever, chills, myalgia, a loss of smell or taste within 14 days prior to a dive session [2]. The clinical criteria for the diagnosis 
Table 1. Classification of COVID-19 [4]

\begin{tabular}{|c|c|c|}
\hline Category & COVID-19 & Symptoms \\
\hline 0 & $\begin{array}{l}\text { Asympto- } \\
\text { matic }\end{array}$ & $\begin{array}{l}\text { No clinical symptoms } \\
\text { Positive screening PCR or antibody test }\end{array}$ \\
\hline 1 & Mild & $\begin{array}{l}\text { Mild clinical symptoms } \\
\text { No health care or outpatient treatment only } \\
\text { Without evidence of hypoxemia, not required supplemental oxygen } \\
\text { Chest X-ray or CT scan normal or not required }\end{array}$ \\
\hline 2 & Moderate & $\begin{array}{l}\text { Clinical symptoms with hypoxemia required supplemental oxygen } \\
\text { Abnormal chest X-ray or CT scan } \\
\text { Hospital treatment not required intubation and assisted mechanical ventilation } \\
\text { Normal cardiac work including normal electrocardiogram and cardiac biomarkers (troponin or CK-MB and BNP) }\end{array}$ \\
\hline 3 & Severe & $\begin{array}{l}\text { Hospital treatment required intubation and assisted mechanical ventilation } \\
\text { Cardiac involvement (abnormal electrocardiogram or echocardiogram, or elevated cardiac biomarkers: } \\
\text { troponin or CK-MB and BNP) } \\
\text { Thromboembolic complications (pulmonary embolism, deep vein thrombosis, or other coagulopathy) }\end{array}$ \\
\hline
\end{tabular}

Table 2. Recommendations for diving fitness health assessment after COVID-19 for commercial and recreational divers [4]

\begin{tabular}{|c|c|c|}
\hline Category & COVID-19 & Tests \\
\hline 0 & $\begin{array}{l}\text { Asympto- } \\
\text { matic }\end{array}$ & $\begin{array}{l}\text { Initial/periodic exam by specialist of hyperbaric medicine/diving doctor } \\
\text { Chest X-ray only, if required by specialist of hyperbaric medicine/diving doctor } \\
\text { No additional testing required }\end{array}$ \\
\hline 1 & Mild & $\begin{array}{l}\text { Initial/periodic exam by specialist of hyperbaric medicine/diving doctor } \\
\text { Spirometry } \\
\text { Chest X-ray (PA and lateral) } \\
\text { Chest CT, if abnormal chest X-ray } \\
\text { If unknown or unsatisfactory exercise tolerance, performing exercise tolerance test with oxygen saturation }\end{array}$ \\
\hline 2 & Moderate & $\begin{array}{l}\text { Initial/periodic exam by specialist of hyperbaric medicine/diving doctor } \\
\text { Spirometry } \\
\text { Chest X-ray (PA and lateral) } \\
\text { Chest CT, if abnormal chest X-ray } \\
\text { Electrocardiogram } \\
\text { Echocardiogram } \\
\text { If unknown or unsatisfactory exercise tolerance, performing exercise tolerance test with oxygen saturation } \\
\text { Investigation and treatment of any other complications by specialist of hyperbaric medicine/diving doctor }\end{array}$ \\
\hline 3 & Severe & $\begin{array}{l}\text { Initial/periodic exam by specialist of hyperbaric medicine/diving doctor } \\
\text { Spirometry } \\
\text { Chest X-ray (PA and lateral) } \\
\text { Chest CT, if abnormal chest X-ray } \\
\text { Electrocardiogram } \\
\text { Repeat cardiac biomarkers (troponin or CK-MB and BNP) to ensure normalization } \\
\text { Echocardiogram } \\
\text { Exercise echocardiogram with oxygen saturation } \\
\text { Investigation and treatment of any other complications by specialist of hyperbaric medicine/diving doctor }\end{array}$ \\
\hline
\end{tabular}

of a COVID-19 infection include at least two of the above symptoms, regardless of the result on a polymerase chain reaction or antibody test for SARS-CoV-2 [10]. Depending on its severity, COVID-19 infection has been classified into four categories (Table 1).

Because COVID-19 is a systemic disease which can involve multiple organs and is likely to cause long-term respiratory and cardiovascular complications which may potentially affect a divers' physical health and diving safety, it is strongly recommended that all divers who have recovered from a COVID-19 infection should undergo a comprehensive diving health assessment before they return to scuba diving. The spectrum of diagnostic tests which are necessary to evaluate an individual's health and fitness to dive will primarily depend on the course and the severity of a COVID-19 infection (Table 2). 
The recommendations of the European Underwater and Baromedical Society (EUBS) and the European Committee for Hyperbaric Medicine (ECHM) [4] with respect to recreational and commercial diving after SARS-CoV-2 pandemic are as follows:

- divers who have tested positive for COVID-19, but were asymptomatic, are recommended to wait at least 1 month before they return to diving;

- divers who had symptoms of a COVID-19 infection, but did not require hospital treatment, should wait at least three months before having their fitness to dive assessed by a hyperbaric medicine/diving doctor;

- divers with a COVID-19 infection who required hospital treatment due to pulmonary symptoms are recommended to wait at least 3 months before having their fitness to dive assessed under the supervision of a hyperbaric medicine/diving doctor; their fitness to dive examination should include a full lung function evaluation, including exercise tolerance test with oxygen saturation and chest CT;

- divers with COVID-19 who required hospital treatment due to cardiac symptoms must wait at least 3 months before they can be examined by a hyperbaric medicine/diving doctor; their fitness to dive assessment should include a full cardiac evaluation including echocardiogram and exercise echocardiogram [11].

Commercial divers who have developed symptoms of a COVID-19 infection and have abnormal test results should be declared 'unfit to dive', while recreational divers should be strongly discouraged from resuming diving activities. This especially applies to individuals with visible damage to the lung parenchyma, air trapping, heart damage or decreased exercise capacity [2]. Asymptomatic individuals who feel perfectly healthy but exhibit subtle radiographic changes and whose spirometry and exercise tolerance test results remain within the lower limit values should refrain from scuba diving for at least 3 months after recovery, until all pathological lesions disappear. Only then can the tests listed in Table 2 be repeated and a patient can be referred for re-examination by a hyperbaric medicine/a dive doctor who will assess whether or not they have any contraindications to scuba diving $[4,11]$.
Conflict of interest: None declared

\section{REFERENCES}

1. Akhmerov A, Marbán E. COVID-19 and the Heart. Circ Res. 2020; 126(10): 1443-1455, doi: 10.1161/CIRCRESAHA.120.317055, indexed in Pubmed: 32252591.

2. Bennett PB, Cronje FJ, Campbell ES. Assessment of Diving Medical Fitness for Scuba Divers and Instructors: Medical, Physical, Physiological, Psychological. Best Publishing Company, USA 2006.

3. Polak SB, Van Gool IC, Cohen D, et al. A systematic review of pathological findings in COVID-19: a pathophysiological timeline and possible mechanisms of disease progression. Mod Pathol. 2020; 33(11): 2128-2138, doi: 10.1038/s41379-020-0603-3, indexed in Pubmed: 32572155.

4. Sadler C, Alvarez Villela M, Van Hoesen K, et al. Diving after SARS-CoV-2 (COVID-19) infection: Fitness to dive assessment and medical guidance. Diving Hyperb Med. 2020; 50(3): 278287, doi: 10.28920/dhm50.3.278-287, indexed in Pubmed: 32957131.

5. Pan F, Ye T, Sun P, et al. Time course of lung changes at chest CT during recovery from coronavirus disease 2019 (COVID-19). Radiology. 2020; 295(3): 715-721, doi: 10.1148/radiol.2020200370.

6. Spagnolo P, Balestro E, Aliberti S, et al. Pulmonary fibrosis secondary to COVID-19: a call to arms? Lancet Respir Med. 2020; 8(8): 750-752, doi: 10.1016/s2213-2600(20)30222-8.

7. Hall WJ, Hall CB. Clinical significance of pulmonary function tests. Alterations in pulmonary function following respiratory viral infection. Chest. 1979; 76(4): 458-465, doi: 10.1378/chest.76.4.458, indexed in Pubmed: 225132.

8. Germonpré P, Balestra C, Pieters T. Influence of scuba diving on asymptomatic isolated pulmonary bullae. Diving Hyperb Med. 2008; 38(4): 206-211, indexed in Pubmed: 22692754.

9. Shi S, Qin Mu, Shen Bo, et al. Association of cardiac injury with mortality in hospitalized patients with COVID-19 in Wuhan, China. JAMA Cardiol. 2020; 5(7): 802-810, doi: 10.1001/jamacardio.2020.0950, indexed in Pubmed: 32211816.

10. Centers for Disease Control and Prevention. Coronavirus disease 2019 (COVID-19) 2020 interim case definition. https://www. cdc.gov/ nndss/conditions/coronavirus-disease-2019-covid-19/ case-definition/2020/ (Approved: April 5, 2020).

11. European Underwater and Baromedical Society, European Committee for Hyperbaric Medicine. EUBS \& ECHM position statement on recreational and professional diving after the Coronavirus disease (COVID-19) outbreak. http://www.echm.org/documents (Accessed: 05 Jan 2021). 